01

\title{
Распределение по пробегам ионов, отраженных от твердого тела: теория и компьютерное моделирование
}

\author{
() А.И. Толмачев, ${ }^{1}$ Л. Форлано ${ }^{2}$ \\ ${ }^{1}$ Российский новый университет, \\ 105005 Москва, Россия \\ 2 Университет Калабрии, \\ 87036 Козенца, Италия \\ e-mail: tolmachev.alex@rambler.ru,forlano@vegachess.com
}

(Поступило в Редакцию 21 сентября 2016 г.)

\begin{abstract}
Задача о распределении по пробегам ионов, отраженных от твердого тела, исследуется теоретически и методами Монте-Карло. Теоретическое рассмотрение основано на решении интегрального уравнения Чандрасекара для трансформанты Лапласа от функции распределения. Получено семейство кривых для распределений по пробегам при различных энергиях ионов и различных отношениях масс ионов и атомов мишени. Разработана программа компьютерного моделирования, использующая приближение парных столкновений и газовую модель твердого тела. Результаты моделирования сопоставляются с теорией и результатами других авторов.
\end{abstract}

DOI: 10.21883/JTF.2017.07.44664.2042

\section{Введение}

Отражение ионов от твердых тел представляет собой один из важных методов исследования структуры поверхности вещества [1]. Когда ионы входят в мишень, они испытывают однократные, кратные и многократные столкновения с атомами мишени. В результате столкновений одни ионы уменьшают свою энергию до некоторого минимального значения, останавливаются внутри мишени и в дальнейшем рассматриваются как имплантированные ионы [2]. Другие ионы возвращаются к поверхности мишени, преодолевают потенциальный барьер, выходят в вакуум и рассматриваются как отраженные ионы. Основными характеристиками отраженных ионов являются коэффициент отражения по числу частиц, коэффициент отражения по энергии, энергетическое и угловое распределения.

Еще одной, менее популярной характеристикой является распределение ионов по пробегам в мишени.

В случае имплантации распределение по пробегам непосредственно связано с распределением имплантированных частиц по глубине мишени. В одной из первых работ [3] авторы рассчитали методом Монте-Карло распределение по глубине ионов калия энергии $4 \mathrm{keV}$ при имплантации в германий. Распределение оказалось монотонно убывающим. В работе [4] методом компьютерного моделирования было получено распределение по глубине мишени ионов гелия с энергиями $10^{2}, 10^{4}$ и $10^{6} \mathrm{eV}$ при имплантации в никель. Все распределения имели куполообразную форму с максимумом, с увеличением энергии ионов максимум отодвигался от поверхности.

В случае отражения под пробегом иона подразумевается полный путь, который он прошел от момента входа в мишень до момента выхода из мишени. Исследование распределения по пробегам представляет интерес по той причине, что это распределение имеет ту же форму, что и энергетическое распределение отраженных ионов. Если распределение по пробегам является монотонно убывающим, энергетическое распределение тоже является монотонным. Если же распределение по пробегам имеет максимум, энергетическое распределение также содержит максимум.

В работе [5] задача о распределении по пробегам решалась в малоугловом приближении, предполагающем, что сечение рассеяния имеет резкий максимум в области малых углов рассеяния. Все полученные распределения имеют максимум. Однако результат [5] справедлив только при больших энергиях ионов. В работах [6,7] показано, что форма распределения по пробегам меняется с энергией, и в двух предельных случаях распределение можно записать в приближенном виде

$$
F(t)=\frac{n}{t} e^{-a t} I_{n}(a t),
$$

где $t$ - пробег иона, $F(t)$ - функция распределения по пробегам, $I_{n}(t)$ - модифицированная функция Бесселя, $a$ - некоторый параметр. В случае малых энергий $n=1$, и распределение по пробегам монотонно убывает. В случае больших энергий $n=2$, распределение по пробегам имеет максимум и при малых пробегах стремится к нулю, $F(0)=0$.

В настоящей работе мы расширяем границы применимости теоретических результатов [5-7]. Рассматривается случай нормального падения ионов на мишень и используется сечение рассеяния, справедливое для любых углов рассеяния и любых энергий ионов. В результате преобразования Лапласа от функции распределения по пробегам задача сводится к решению интегрального уравнения Чандрасекара для трансформанты Лапласа. 
После решения интегрального уравнения обратное преобразование Лапласа дает семейство распределений по пробегам для различных энергий ионов и различных отношений масс ионов и атомов мишени.

Для проверки теоретических результатов мы разработали программу компьютерного моделирования PAOLA. Программа использует приближение парных столкновений, газовую модель твердого тела для расчета расстояния между двумя последовательными столкновениями и применима для расчета отражения ионов от аморфных мишеней. Проведено прямое сравнение теории и результатов компьютерного моделирования.

\section{Интегральное уравнение}

Мы решаем следующую задачу. Ионы с атомным номером $Z_{1}$, массой $M_{1}$ и энергией $E_{0}$ падают по нормали на поверхность полубесконечной мишени, состоящей из атомов с порядковым номером $Z_{2}$ и массой $M_{2}$. В результате упругих столкновений с атомами мишени ионы меняют направления движения, возвращаются к поверхности и выходят в вакуум. Требуется найти функцию $F(t)$, выражающую распределение отраженных ионов по пробегам в мишени, проинтегрированное по всем углам выхода.

В результате применения преобразования Лапласа

$$
R(s)=\int_{0}^{\infty} F(t) e^{-s t} d t
$$

поставленная задача сводится к решению нелинейного интегрального уравнения Чандрасекара $[8,9]$

$$
\begin{aligned}
& \left(\mu+\mu_{0}\right) R\left(\mu, \mu_{0}\right)=p\left(\mu,-\mu_{0}\right)+\mu \int_{0}^{1} p\left(\mu_{0}, \mu^{\prime}\right) R\left(\mu^{\prime}, \mu\right) d \mu^{\prime} \\
& +\mu_{0} \int_{0}^{1} p\left(\mu, \mu^{\prime}\right) R\left(\mu^{\prime}, \mu_{0}\right) d \mu^{\prime}+\mu \mu_{0} \int_{0}^{1} \int_{0}^{1} p\left(\mu^{\prime},-\mu^{\prime \prime}\right) \\
& \quad \times R\left(\mu, \mu^{\prime}\right) R\left(\mu^{\prime \prime}, \mu_{0}\right) d \mu^{\prime} d \mu^{\prime \prime} .
\end{aligned}
$$

В уравнении (3) через $R\left(\mu, \mu_{0}\right)$ обозначена функция отражения, зависящая от угла падения ионов на поверхность мишени $\theta_{0}\left(\mu_{0}=\cos \theta_{0}\right)$ и угла выхода отраженных ионов $\theta(\mu=\cos \theta)$,

$$
p\left(\mu, \mu^{\prime}\right)=\frac{w_{0}}{2} \sigma\left(\mu, \mu^{\prime}\right), \quad w_{0}=\frac{1}{1+s},
$$

где $\sigma\left(\mu, \mu^{\prime}\right)$ - сечение рассеяния, усредненное по азимутальному углу, и $w_{0} \leq 1-$ параметр поглощения (альбедо).

Уравнение (3) несколько отличается от первоначального уравнения Чандрасекара. Во-первых, учитывая нормальное падение ионов на мишень, мы усреднили уравнение Чандрасекара по азимутальному углу. И вовторых, вместо функции рассеяния $S\left(\mu, \mu_{0}\right)$ была введена функция отражения $R\left(\mu, \mu_{0}\right)=\mu \mu_{0} S\left(\mu, \mu_{0}\right)$, позволяющая исключить особенности под знаками интегралов.

Обычным методом решения уравнения (3) является выбор некоторого упрощенного сечения рассеяния, допускающего аналитическое рассмотрение $[8,10,11]$. В настоящей работе мы используем сечение рассеяния общего вида и решаем интегральное уравнение (3) численно, методом последовательных приближений.

После вычисления функции отражения коэффициент отражения по числу частиц определяется интегрированием

$$
R_{N}\left(w_{0}\right)=\int_{0}^{1} \mu R\left(\mu, \mu_{0}\right) d \mu,
$$

уравнение (4) дает трансформанту $R(s)=R_{N}\left[(1+s)^{-1}\right]$, и обратное преобразование Лапласа приводит к функции распределения по пробегам.

\section{Сечение рассеяния}

Мы воспользуемся атомным потенциалом Менсинг, для которого известно аналитическое решение задачи рассеяния как в квантово-механическом [12], так и в классическом приближении $[13,14]$. Для потенциала Менсинг энергия взаимодействия двух зарядов $Z_{1} e$ и $Z_{2} e$ на расстоянии $r$ равна

$$
U(r)=\frac{Z_{1} Z_{2} e^{2}}{4 \pi \varepsilon_{0}}\left(\frac{1}{r}-\frac{1}{r_{0}}\right) \quad \text { при } \quad r \leq r_{0} .
$$

$U(r)=0$ при $r \geq r_{0}$, где $r_{0}-$ радиус отсечки, $e-$ заряд электрона, $\varepsilon_{0}$ - электрическая постоянная. Потенциал (6) является весьма грубым приближением к истинному атомному потенциалу. Поэтому постоянные величины в уравнении (6) обычно рассматриваются как подгоночные параметры, которые выбираются путем согласования с одним из стандартных потенциалов по значению логарифмической производной в точке наибольшего сближения. Примеры согласования с обратно квадратичным и экранированным кулоновским потенциалами приведены в работе [13].

Методами классической теории рассеяния [15] получаем зависимость угла рассеяния в системе центра масс $\omega$ от прицельного расстояния $\rho$ :

$$
\cos \omega=\frac{(1+2 \eta) b^{2}-\eta}{b^{2}+\eta} .
$$

Здесь $b=\rho / r_{0}, \varepsilon=E / E_{a t}-$ приведенная энергия,

$$
E_{a t}=\left(1+\frac{M_{1}}{M_{2}}\right) \frac{Z_{1} Z_{2} e^{2}}{4 \pi \varepsilon_{0} r_{0}}, \quad \eta=\frac{1}{4 \varepsilon(1+\varepsilon)},
$$

$E_{a t}-$ единица энергии, $\eta-$ параметр экранирования. Из (7) находим

$$
b^{2}=\frac{\eta(1+\cos \omega)}{1+2 \eta-\cos \omega}
$$


и дифференциальное сечение рассеяния в системе центра масс, нормированное на единицу:

$$
d \sigma=2 b d b=\frac{2 \eta(1+\eta) \sin \omega d \omega}{(1+2 \eta-\cos \omega)^{2}} .
$$

Угол рассеяния в лабораторной системе координат $\Omega$ связан с углом $\omega$ :

$$
\cos \Omega=\frac{A+\cos \omega}{\sqrt{1+2 A \cos \omega+A^{2}}},
$$

где $A=M_{1} / M_{2} \leq 1-$ отношение массы иона к массе атома мишени. Из (10), (11) вычисляем дифференциальное сечение рассеяния в лабораторной системе координат:

$$
\frac{d \sigma}{\sin \Omega d \Omega}=\frac{2 \eta(1+\eta)\left(2 A \cos \Omega+\frac{1+A^{2} \cos 2 \Omega}{\sqrt{1-A^{2} \sin ^{2} \Omega}}\right)}{\left(1+2 \eta+A \sin ^{2} \Omega-\cos \Omega \sqrt{1-A^{2} \sin ^{2} \Omega}\right)^{2}} .
$$

Если полярный и азимутальный углы $\left(\theta_{1}, \varphi_{1}\right)$ задают направление скорости иона до столкновения, а углы $\left(\theta_{2}, \varphi_{2}\right)$ - после столкновения и если $\mu_{1}=\cos \theta_{1}$, $\mu_{2}=\cos \theta_{2}$, то угол $\Omega$ определяется из уравнения

$$
\cos \Omega=\mu_{1} \mu_{2}+\sqrt{1-\mu_{1}^{2}} \sqrt{1-\mu_{2}^{2}} \cos \left(\varphi_{1}-\varphi_{2}\right) .
$$

\section{Теоретические результаты}

Мы провели численное решение уравнения (3) для сечения рассеяния (12), усредненного по азимутальному углу с учетом (13). Интегрирование (5) дало зависимость коэффициента отражения от параметра поглощения $w_{0}$ и приведенной энергии $\varepsilon_{0}=E_{0} / E_{a t}$. Результаты расчетов приведены в табл. 1 и 2 для двух предельных случаев: $A=0$ (масса иона $M_{1}$ намного меньше массы атома мишени $M_{2}$ ) и $A=1$ (массы ионов и атомов мишени равны).

Таблица 1. Функция $R_{N}\left(w_{0}\right)$ при $A=0$ для различных энергий

\begin{tabular}{c|c|c|c|c|c|c}
\hline$w_{0}$ & $\varepsilon_{0}=0$ & $\varepsilon_{0}=1$ & $\varepsilon_{0}=2$ & $\varepsilon_{0}=3$ & $\varepsilon_{0}=4$ & $\varepsilon_{0}=5$ \\
\hline 0.00 & 0.00000 & 0.00000 & 0.00000 & 0.00000 & 0.00000 & 0.00000 \\
0.10 & 0.01639 & 0.00295 & 0.00111 & 0.00057 & 0.00035 & 0.00023 \\
0.20 & 0.03523 & 0.00683 & 0.00257 & 0.00131 & 0.00079 & 0.00053 \\
0.30 & 0.05721 & 0.01204 & 0.00456 & 0.00231 & 0.00138 & 0.00092 \\
0.40 & 0.08336 & 0.01927 & 0.00739 & 0.00373 & 0.00222 & 0.00146 \\
0.50 & 0.11523 & 0.02968 & 0.01164 & 0.00586 & 0.00345 & 0.00226 \\
0.60 & 0.15541 & 0.04544 & 0.01849 & 0.00934 & 0.00547 & 0.00355 \\
0.70 & 0.20868 & 0.07103 & 0.03074 & 0.01577 & 0.00921 & 0.00592 \\
0.80 & 0.28525 & 0.11740 & 0.05624 & 0.03021 & 0.01786 & 0.01144 \\
0.90 & 0.41495 & 0.22112 & 0.12671 & 0.07660 & 0.04857 & 0.03220 \\
0.95 & 0.53554 & 0.34384 & 0.22946 & 0.15720 & 0.11010 & 0.07871 \\
0.99 & 0.75272 & 0.61910 & 0.51578 & 0.43321 & 0.36594 & 0.31051 \\
1.00 & 1.00000 & 1.00000 & 1.00000 & 1.00000 & 1.00000 & 1.00000
\end{tabular}

Таблица 2. Функция $R_{N}\left(w_{0}\right)$ при $A=1$ для различных энергий

\begin{tabular}{c|c|c|c|c|c|c}
\hline$w_{0}$ & $\varepsilon_{0}=0$ & $\varepsilon_{0}=1$ & $\varepsilon_{0}=2$ & $\varepsilon_{0}=3$ & $\varepsilon_{0}=4$ & $\varepsilon_{0}=5$ \\
\hline 0.00 & 0.00000 & 0.00000 & 0.00000 & 0.00000 & 0.00000 & 0.00000 \\
0.10 & 0.00019 & 0.00001 & 0.00000 & 0.00000 & 0.00000 & 0.00000 \\
0.20 & 0.00093 & 0.00005 & 0.00001 & 0.00000 & 0.00000 & 0.00000 \\
0.30 & 0.00260 & 0.00014 & 0.00002 & 0.00001 & 0.00000 & 0.00000 \\
0.40 & 0.00584 & 0.00037 & 0.00006 & 0.00002 & 0.00001 & 0.00000 \\
0.50 & 0.01179 & 0.00087 & 0.00015 & 0.00004 & 0.00002 & 0.00001 \\
0.60 & 0.02264 & 0.00206 & 0.00037 & 0.00010 & 0.00004 & 0.00002 \\
0.70 & 0.04302 & 0.00508 & 0.00101 & 0.00029 & 0.00011 & 0.00005 \\
0.80 & 0.08445 & 0.01410 & 0.00329 & 0.00100 & 0.00038 & 0.00017 \\
0.90 & 0.18566 & 0.05127 & 0.01656 & 0.00609 & 0.00252 & 0.00116 \\
0.95 & 0.31103 & 0.12548 & 0.05478 & 0.02539 & 0.01243 & 0.00642 \\
0.99 & 0.59864 & 0.40239 & 0.27793 & 0.19491 & 0.13827 & 0.09924 \\
1.00 & 1.00000 & 1.00000 & 1.00000 & 1.00000 & 1.00000 & 1.00000
\end{tabular}

В случае $A=0$ и при малых энергиях ионов (колонка $\varepsilon_{0}=0$ в табл. 1$)$, сечение изотропно и известно точное решение

$$
R_{N}\left(w_{0}\right)=1-\sqrt{1-w_{0}} H\left(w_{0}\right)
$$

где $H\left(w_{0}\right)$ - функция Чандрасекара [8]. Уравнение (14) использовалось для проверки состоятельности метода и его точности. Все цифры во колонке $\varepsilon_{0}=0$ совпали со своими точными значениями. Последующие колонки обобщают изотропное решение на анизотропный случай.

Используя табл. 1 и 2 и учитывая соотношения (4), мы выполнили обратное преобразование Лапласа и получили распределение отраженных ионов по пробегам. Распределения изображены на рис. 1 для случаев $A=0$ и $A=1$ и различных энергий ионов. Пробег измерялся в единицах транспортной длины $t_{t r}=1 / \sigma_{t r}$, где транспортное сечение находится из (12):

$$
\sigma_{t r}= \begin{cases}2 \eta\left[(1+\eta) \ln \left(1+\frac{1}{\eta}\right)-1\right] & \text { при } A=0, \\ \eta\left(\frac{\sqrt{1+\eta}}{2} \ln \frac{\sqrt{1+\eta}+1}{\sqrt{1+\eta}-1}-1\right) & \text { при } A=1 .\end{cases}
$$

В случае $A=0$ значение функции распределения при нулевом пробеге $F(0)$ определяется ионами, испытавшими ровно одно столкновение. Учитывая только первое слагаемое в правой части (3), из (4) и (5) получаем

$$
F(0)=\frac{1}{2}\left[1-\left(1+\frac{1}{\eta}\right) \ln \left(1+\frac{\eta}{1+\eta}\right)\right] \text { при } A=0 .
$$

Из рис. $1, a$ мы видим, что в случае $A=0$ и при малых энергиях ионов распределение по пробегам является монотонно убывающим. При увеличении энергии на распределении появляется максимум. При дальнейшем росте энергии высота максимума увеличивается, а его полуширина уменьшается. На рис. $1, b$ показаны распределения по пробегам в случае $A=1$. Распределения при 

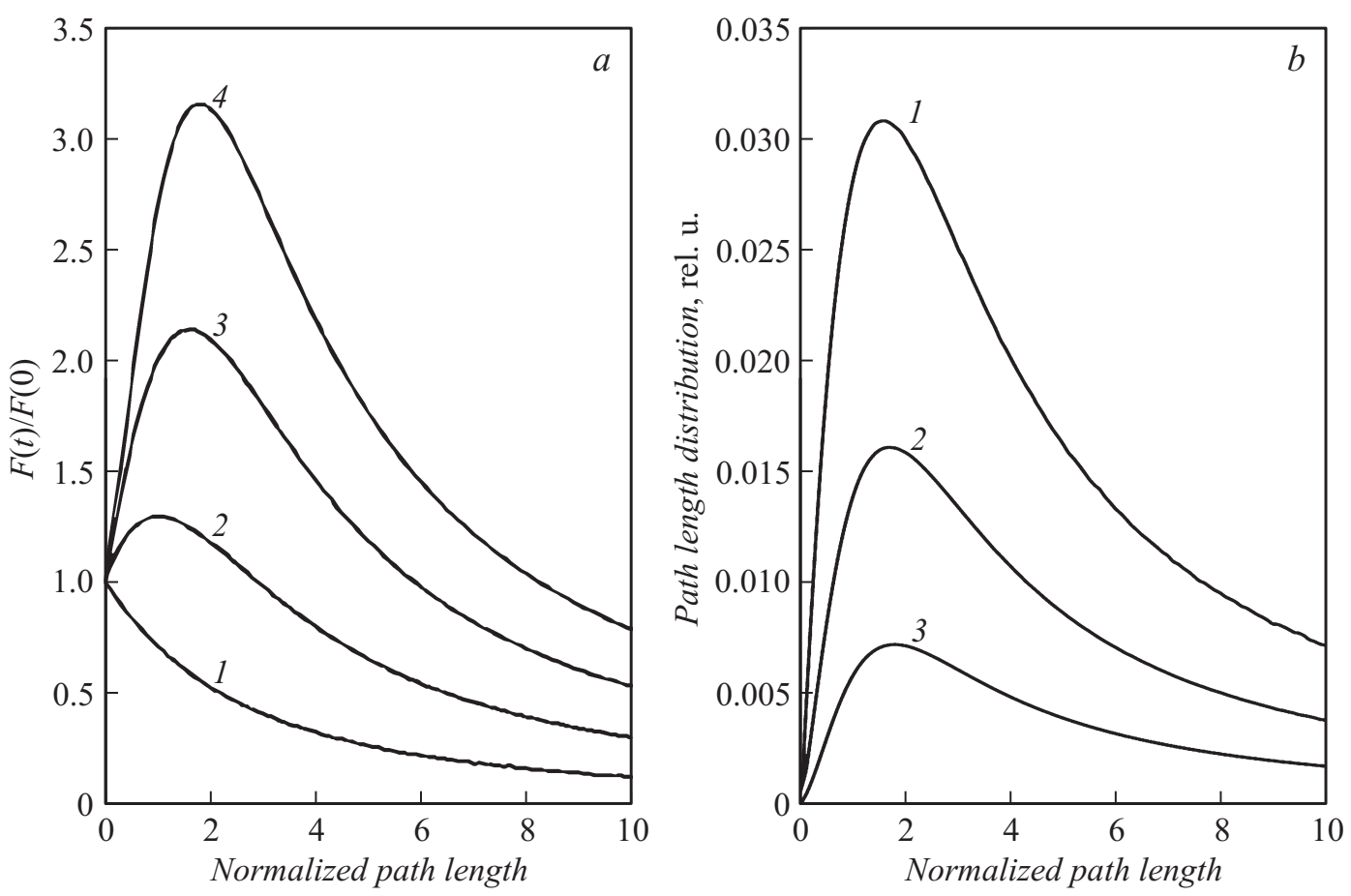

Рис. 1. Результаты теории для функции распределения отраженных ионов по пробегам в мишени. $a-M_{1} \ll M_{2}: \varepsilon_{0}=0(1)$, $1(2), 3(3), 7(4) ; b-M_{1}=M_{2}: \varepsilon_{0}=0(1), 0.5(2), 1.5$ (3).

$A=0$ и $A=1$ демонстрируют качественное различие. В первом случае все распределения начинаются с конечного значения (16) при $t=0$, так как всегда имеются ионы, вышедшие из мишени после одного столкновения. Во втором случае все распределения начинаются с нуля при $t=0$, так как для выхода из мишени ион должен испытать по крайней мере два столкновения. Отметим, что приближенная формула (1) хорошо описывает оба предельных случая не только качественно, но и количественно.

\section{Результаты моделирования}

Для проверки результатов теории мы создали программу компьютерного моделирования PAOLA, названную по имени города, в котором проживает один из авторов. Алгоритм решения основан на формулах $(17)-(21)$, приведенных ниже.

В процессе моделирования датчик генерирует три случайных числа $R_{1}, R_{2}, R_{3}$ в интервале от 0 до 1 . Пробег иона между двумя последовательными столкновениями равен

$$
\lambda=-\lambda_{0} \ln R_{1}
$$

где $\lambda_{0}-$ средняя длина свободного пробега. Из (7) находим полярный угол рассеяния в системе центра масс,

$$
\cos \omega=\frac{(1+2 \eta) R_{2}-\eta}{R_{2}+\eta}
$$

а из (11) - полярный угол рассеяния в лабораторной системе координат. Азимутальный угол рассеяния равен

$$
\psi=2 \pi R_{3} .
$$

Если полярный угол $\theta_{n}$ определяет направление скорости иона до столкновения, а угол $\theta_{n+1}-$ после столкновения, то эти углы связаны формулой сферической геометрии

$$
\cos \theta_{n+1}=\cos \theta_{n} \cos \Omega-\sin \theta_{n} \sin \Omega \cos \psi
$$

И наконец, отношение энергии иона после столкновения $E_{n+1}$ к его энергии до столкновения $E_{n}$ определяется из законов сохранения энергии и импульса:

$$
\frac{E_{n+1}}{E_{n}}=\left(\frac{A \cos \Omega+\sqrt{1-A^{2} \sin ^{2} \Omega}}{1+A}\right)^{2} .
$$

Используя $10^{7}$ пробных частиц, мы воспроизвели заново табл. 1 и 2, но получили при этом некоторые отклонения, зависящие от начального значения в генераторе случайных чисел. Наибольшая погрешность не превысила 1\% для всех 156 чисел в таблицах.

Результаты моделирования распределения отраженных ионов по пробегам представлены на рис. 2. Гистограммы показывают хорошее согласие с теоретическими кривыми на рис. 1 во всем диапазоне пробегов, кроме начальной области $t \approx 0$. Это расхождение является следствием интегрального характера представления функций в виде гистограмм. Увеличение точности отображения 

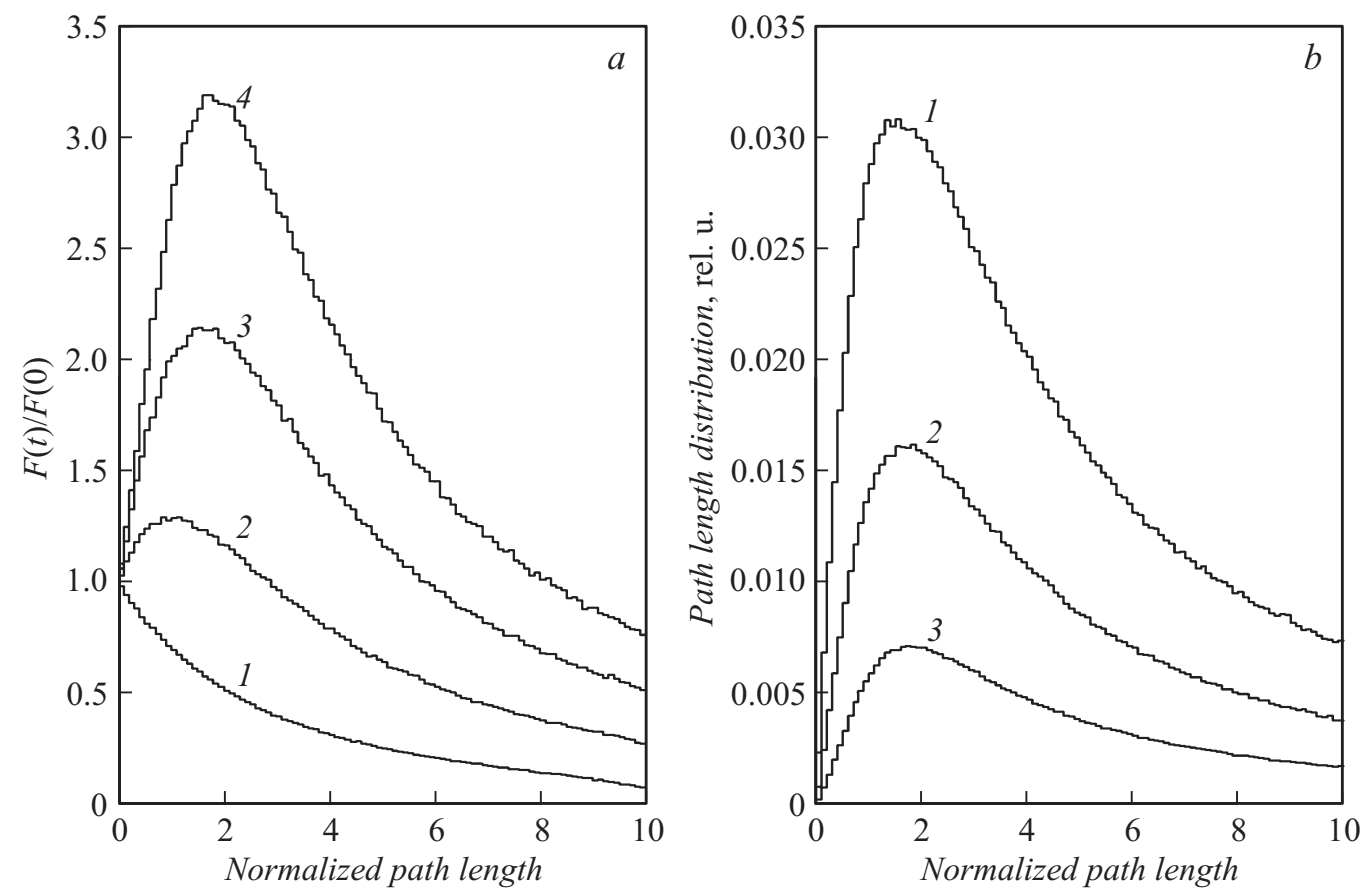

Рис. 2. Результаты моделирования для функции распределения отраженных ионов по пробегам в мишени. $a-M_{1} \ll M_{2}$ : $\varepsilon_{0}=0(1), 1(2), 3(3), 7(4) ; b-M_{1}=M_{2}: \varepsilon_{0}=0$ (1), $0.5(2), 1.5$ (3).

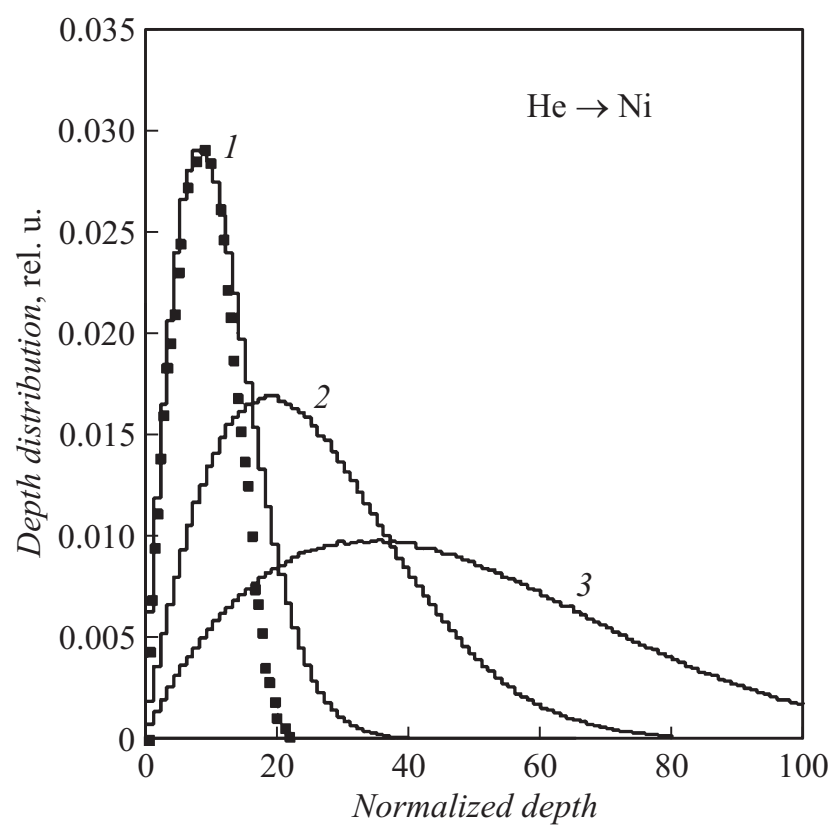

Рис. 3. Результаты моделирования для распределения по глубине мишени ионов гелия, имплантированных в никель. Энергии ионов 10 (1), 30 (2), $50 \mathrm{keV}$ (3). Маркеры моделирование [4] для энергии $10 \mathrm{keV}$.

предельных значений при $t \rightarrow 0$ требует дополнительного исследования.

В завершение работы мы решили задачу, которая уже моделировалась ранее программой TRIM - имплантация гелия в никелевую мишень. В качестве радиуса отсечки был выбран радиус экранирования Фирсова, глубина мишени измерялась в длинах свободного пробега, энергия остановки составляла $10 \mathrm{eV}$. Рис. 3 показывает распределение имплантированных ионов по глубине их проникновения в мишень. На всех распределениях наблюдается максимум. С увеличением энергии ионов максимум отодвигается от поверхности и размывается. При энергии $10 \mathrm{keV}$ распределение хорошо согласуется с результатами [4]. Некоторое расхождение в полуширине распределений можно объяснить использованием разных атомных потенциалов.

\section{Заключение}

Решена задача о распределении ионов, отраженных от твердого тела, по пробегам в мишени. При рассмотрении столкновений ионов с атомами мишени использовано сечение рассеяния, справедливое без ограничений для любых энергий и углов рассеяния ионов, что позволило обобщить результаты предыдущих теорий. Показано, что форма распределения меняется качественно при изменении энергии и массы ионов. Распределение по пробегам может быть как монотонно убывающим, так и куполообразным, с максимумом.

Разработана программа компьютерного моделирования, использующая приближение парных столкновений и газовую модель твердого тела. Проведено прямое сопоставление результатов моделирования с теоретическими результатами, что позволило сделать выводы о точности и некоторых недостатках методики Монте-Карло. 
Результаты моделирования общепризнанных компьютерных программ - MARLOWE, TRIM, SRIM и др. могут быть сопоставлены с результатами эксперимента, но не с теорией. Настоящая работа устраняет этот пробел.

Обобщение алгоритма решения на случай других атомных потенциалов не составляет труда. Для потенциала (6) угол рассеяния выражается через прицельный параметр аналитической формулой (7), что позволяет использовать очень большое число пробных частиц. В случае других потенциалов угол рассеяния не может быть представлен аналитической формулой, для каждого столкновения его требуется вычислять численно, путем интегрирования. Для того чтобы сохранить прежнее время расчета, приходится уменьшать число пробных частиц на два или три порядка.

\section{Список литературы}

[1] Машкова E.C., Молчанов В.А. Применение рассеяния ионов для анализа твердых тел. М.: Энергоатомиздат, 1995. $175 \mathrm{c}$

[2] Комаров Ф.Ф. Ионная имплантация в металлы. М.: Металлургия, 1990. $216 \mathrm{c.}$

[3] Бредов М.М., Ланг И.Г., Окунева Н.М. // ЖТФ. 1958. Т. 28. Вып. 2. С. 252.

[4] Eckstein W. Computer Simulation of Ion-Solid Interactions. Berlin: Springer-Verlag, 1991. 296 p.

[5] Рязанов М.И., Тилинин И.С. Исследование поверхности по обратному рассеянию частиц. М.: Энергоатомиздат, 1985. $152 \mathrm{c}$

[6] Толмачев А.И. // ЖТФ. 1986. Т. 56. Вып. 7. С. 1297.

[7] Falcone G., Forlano L., Tolmachev A.I. // Phys. Rev. B. 1999. Vol. 60. P. 6352.

[8] Chandrasekhar S. Radiative Transfer. Oxford: Clarendon Press, 1950. $393 \mathrm{p}$.

[9] Толмачев А.И., Форлано Л. // Поверхность. 2017 (в печати).

[10] Borodyansky S., Tougaard S. // Surf. Interfac. Analys. 1995. Vol. 23. P. 689.

[11] Remizovich V.S., Radkevich A.V. // Laser Phys. 2000. Vol. 10. P. 560.

[12] Mensing L. // Zeitshrift fur Physik. 1927. B. 45. S. 158.

[13] Leibfried G., Oen O.S. // J. Appl. Phys. 1962. Vol. 33. P. 2257.

[14] Толмачев А.И. // Поверхность. 1992. Вып. 8. С. 27.

[15] Ландау Л.Д., Лифиии Е.М. Механика. Издание 5-е, стереотипное. М.: Физматлит, 2012. 224 с. 